\title{
EXPERIMENTAL AERODYNAMIC ASSESSMENT AND EVALUATION OF AN AGILE HIGHLY SWEPT AIRCRAFT CONFIGURATION
}

\author{
K.C. Huber, A. Schütte \\ DLR, Institute of Aerodynamics and Flow Technology, \\ Lilienthalplatz 7, D-38108 Braunschweig, Germany \\ M. Rein \\ DLR, Institute of Aerodynamics and Flow Technology, \\ Bunsenstraße 10, D-37073 Göttingen, Germany \\ T. Löser \\ DNW, Niedergeschwindigkeits-Windkanal (NWB) \\ Lilienthalplatz 7, D-38108 Braunschweig, Germany
}

\begin{abstract}
Extensive experimental and numerical investigations on a highly swept generic unmanned combat aerial vehicle (UCAV) configuration of lambda type with a variable leading edge contour have been conducted. Within these investigations it was shown that the flow field is dominated by complex vortex systems including vortex-to-vortex and vortex-to-boundary layer interactions. The vortex dominated flow field has a strong nonlinear influence on the aerodynamic behavior of the configuration. Hence, the controllability aspect is demanding and poses a real challenge in the design of this kind of configuration. Especially the dimensioning of the control surfaces for the lateral- and longitudinal stability aspects of tailless configurations of low aspect ratio and high leading edge sweep poses a challenging task, which is not yet solved.

The present paper reviews the experimental aspects of the investigations. In order to understand the problem of lacking lateral- and longitudinal stability for these kind of configuration, experiments in the subsonic and transonic flow regime have been conducted for the SACCON configuration, which has a leading edge sweep of $53^{\circ}$ in order to assess the control surface effectiveness of conventional trailing edge control devices. These investigations were undertaken as part of the internal DLR projects FaUSST and Mephisto.
\end{abstract}

\section{NOMENCLATURE}

\begin{tabular}{|c|c|c|}
\hline B & $=$ & wing span [m] \\
\hline $\mathrm{C}_{\mathrm{L}}$ & $=$ & lift coefficient [-] \\
\hline$C_{D}$ & $=$ & drag coefficient [-] \\
\hline $\mathrm{C}_{Y}$ & $=$ & side force coefficient [-] \\
\hline $\mathrm{C}_{1}$ & $=$ & rolling moment coefficient [-] \\
\hline $\mathrm{C}_{\mathrm{m}}$ & $=$ & pitching moment coefficient [-] \\
\hline $\mathrm{C}_{\mathrm{n}}$ & $=$ & yawing moment coefficient $[-]$ \\
\hline CS & $=$ & control surface \\
\hline$c_{\text {ref }}$ & $=$ & reference chord length [m] \\
\hline $\mathrm{C}_{\mathrm{r}}$ & $=$ & root chord [m] \\
\hline $\mathrm{C}_{\mathrm{MRP}}$ & $=$ & chord length location of MRP [m] \\
\hline L.E. & $=$ & leading edge \\
\hline LIB & $=$ & left hand inboard \\
\hline LOB & $=$ & left hand outboard \\
\hline M & $=$ & Mach number [-] \\
\hline MRP & $=$ & Moment Reference Point \\
\hline $\operatorname{Re}$ & $=$ & Reynolds number, based on $\mathrm{c}_{\text {ref }}$ \\
\hline RIB & $=$ & right hand inboard \\
\hline ROB & $=$ & right hand outboard \\
\hline $\mathrm{s}$ & $=$ & wing half-span $[\mathrm{m}]=0.5 \cdot \mathrm{b}$ \\
\hline miar & & \\
\hline & & \\
\hline
\end{tabular}
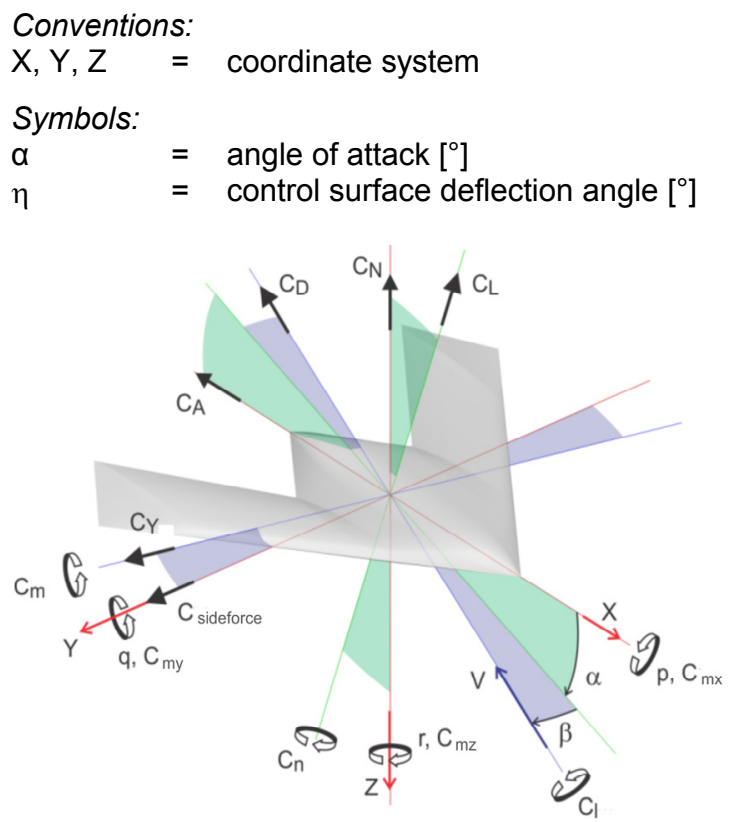

FIGURE 1. Coordinate system, see Vicroy et al. [1] 


\section{INTRODUCTION}

In recent years increased attention has been drawn to research and design of agile UCAVs, which need to fulfill requirements for long distance flight and stealth characteristics but also need to maintain high maneuverability throughout a wide Mach number range along the flight envelope. These explicitly imposed performance and endurance requirements lead to tailless plan forms based on the idea of flying wings, with outline shapes varying between a pure delta, lambda or diamond wing.

The geometric constraints of these kind of configurations impose possible control concepts to be applied at the wing leading- and trailing edges or on the upper configuration surface, by means of spoiler. Large geometrical control surface deflections could however be constrained by stealth requirements and hence a possible alternative control concept by means of thrust vectoring could be applied [2]

Throughout a series of internal DLR projects as well as international research groups in the framework of the Applied Vehicle Panel (AVT) of the NATO ${ }^{1}$ Science and Technology Organizations (STO) ${ }^{2}$ a variety of different highly swept aircraft configurations with round leading edge radius were experimentally and numerically analyzed. The latest configuration shape considered, which is also the main subject matter of the here presented experiments, is a flying wing configuration of lambda-wing type with a variable leading edge contour along the span, and accommodating a leading edge sweep of $53^{\circ}$. The configuration is known as the SACCON (Stability And Control CONfiguration).

The SACCON baseline configuration, without control surfaces, was subject of extensive experimental and numerical research as part of the DLR internal project UCAV-2010 and the international research group RTO/AVT-161 [3]. For this research, two wind tunnel models had been built, the low speed wind tunnel model of same name, and a high speed model, the DLR-F17E [4]. One of the main objectives of these two projects was to improve the ability to accurately predict both static and dynamic stability for these kinds of configurations using RANS $^{3}$ methods [5]. The conducted experiments were used to establish a highly accurate experimental database for code validation purposes of existent computational fluid dynamics (CFD) solvers.

The flow topology and the developed best practices to model the flow as well as the aerodynamic behavior of the baseline configuration are now well understood [6].

The international successor group AVT-201 as well as the two internal DLR successor projects FaUSST and Mephisto took the topic further to include controllability aspects. The objective of these research groups was to use the understanding of the flow structure to implement a first control concept, designed using best practices developed in the aforementioned predecessor projects.

Within a first control concept consideration, the generic UCAV configuration SACCON has been equipped with

\footnotetext{
${ }^{1}$ North Atlantic Treaty Organisation

${ }^{2}$ Up to Sept. 2012: Research and Technology Organization

${ }^{3}$ Reynolds Averaged Navier-Stokes
}

trailing edge control devices, with their hinge lines passing parallel to the trailing edge.

The aim is to understand and to be able to numerically model the underlying flow structure altered by the deflection of the trailing edge devices and their resulting influences on the aerodynamic behavior, especially to reach an acceptable controllability in lateral- and longitudinal axis direction. It is aimed to develop a hybrid database, consisting of experimental data paired with numerical results, including control surface derivatives. This database forms the basis for an aerodynamic replacement model for stability and control analyses to develop a novel mission driven flying wing configuration.

For the test on the generic UCAV configuration SACCON with the first control concept a new low speed wind tunnel model, the DLR-F19 was built to incorporate control surfaces. Additionally, the high speed wind tunnel model DLR-F17E has been modified to include control surfaces as well. The DLR-F19 and the high speed wind tunnel model DLR-F17E serve as experimental demonstrators within this here presented work. These configurations have been tested in the low speed wind tunnel facility (DNWNWB) Braunschweig and the transonic wind tunnel facility (DNW-TWG) Göttingen.

The control surfaces considered are simple up- and downward deflected trailing edge control surfaces with an angle of deflection of up to $\eta= \pm 20^{\circ}$. The aim of this work is to identify the effectiveness of conventional static trailing control surfaces applied to the generic lambda wing configuration SACCON at low to transonic speeds and an angle of attack range of $\alpha=0^{\circ}-24^{\circ}$.

\section{EXPERIMENTS}

\subsection{DLR-F19 Low Speed Model}

Static low speed tests have been conducted in the low speed testing facility of the German-Dutch Wind Tunnels, DNW-NWB ${ }^{4}$, located on the premises of the DLR in Braunschweig, using the DLR-F19 wind tunnel model.

The DNW-NWB is a closed circuit low speed wind tunnel of atmospheric type. For the tests described in this paper, the closed test section was used at a test velocity of $\mathrm{M}=$ 0.15 and a Reynolds number of $\operatorname{Re}=1.6 \cdot 10^{6}$. Further information concerning the DNW-NWB and its measurement techniques can be found on the DNW-NWB website [7], in Rein et al. [8] and in Bergmann et al.[9].

The DLR-F19 model dimensions are depicted in FIGURE 2. The moment reference point (MRP) is located at $(x / y / z)$ $=(0.6 \mathrm{~m} / 0 / 0)$.

The model has a weight of approximately $10 \mathrm{~kg}$ and is of modular set-up, i.e. the control surface geometries can be exchanged and refitted. The control surfaces are nonadjustable; hence for each angle of deflection a control surface geometry is existent. The hinge line of the considered control surfaces is located parallel to the trailing edge at $\mathrm{c}_{\text {ref }}=75 \%$.

In order to fix transition for the DLR-F19 it was chosen to prepare the leading edge with a three-dimensional artificial transition, by means of a corundum grit [10]. A removable

\footnotetext{
${ }^{4}$ Niedergeschwindigkeits-Windkanal Braunschweig
} 
latex based paint is used to fix the transition and hence it is possible to remove and reapply the transition without damaging the surface of the wind tunnel model. The grit was applied to approximately the first $25 \mathrm{~mm}$ at the nose to $10 \mathrm{~mm}$ at the wing tip along both, the upper and lower surface of the configurations leading edge. In subsequent infrared thermography investigations it was shown that a fixed transition line is present and hence a fully turbulent flow over the upper wing surface was assumed [10].

The DLR-F19 is equipped with a total of 165 surface pressure ports, consisting of 115 static pressure sensors and 12 Kulites, located in four lines of intersection, at $\mathrm{x} / \mathrm{c}_{\mathrm{r}}=0.2,0.45$ and $\mathrm{y} / \mathrm{s}=0.67$ and 0.89 , perpendicular to the leading edge, respectively. These surface pressure collection planes are depicted in FIGURE 2.

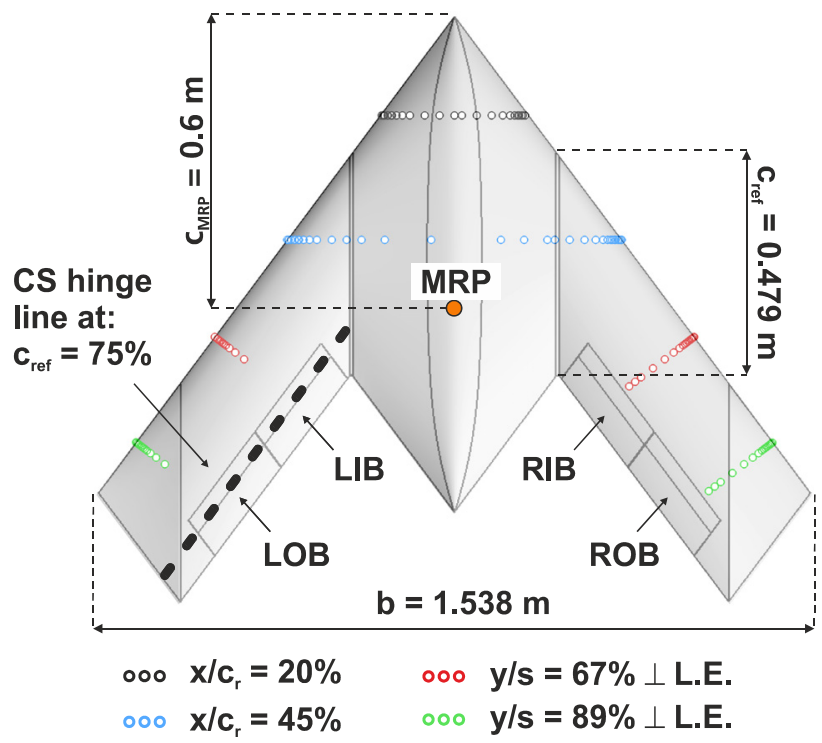

FIGURE 2. DLR-F19 model dimensions, location of control surfaces and instrumentation.

An internal balance is used to collect the force and moment data of the wind tunnel model configuration, which is a 6-component strain gage balance of type Emmen-1966 , placed within the wind tunnel model. Throughout the experimental investigation the model was mounted by a belly sting support from the Moment Positioning Mechanism (MPM) Steward platform. This belly sting arrangement is a yaw link support with a crank angle of $15^{\circ}$, depicted in FIGURE 3 . The $15^{\circ}$ crank angle is applied to test an angle of attack range between $0^{\circ}-30^{\circ}$. The model within its wind tunnel arrangement can be seen in FIGURE 4

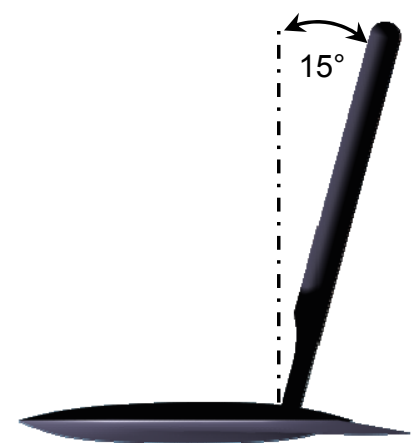

FIGURE 3.DNW-NWB MPM belly sting arrangement - yaw link support with $15^{\circ}$ crank angle.

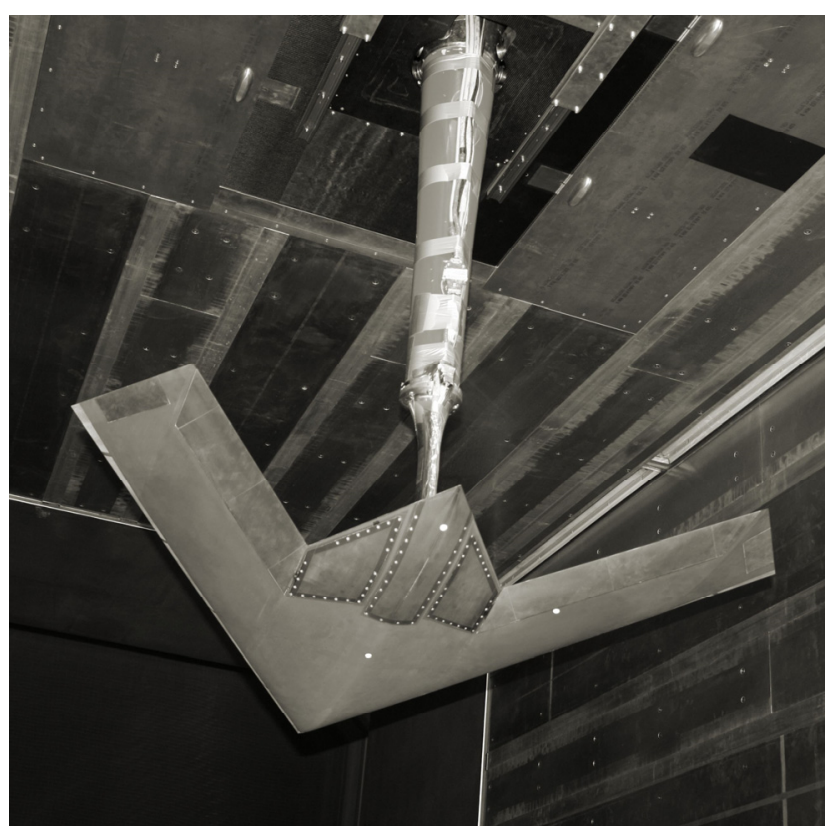

FIGURE 4. DLR-F19 wind tunnel mounting arrangement of the belly $15^{\circ}$ yaw link support in the DNW-NWB.

\subsection{DLR-F17E High Speed Model}

Static high speed test cases have been conducted in the high speed testing facility of the DNW TWG, located on the premises of the DLR in Göttingen, using the DLRF17E wind tunnel model. The DNW-TWG is a closed circuit, continuous tri-sonic wind tunnel. For the high speed static tests a perforated test section was used at a test velocity ranging from $\mathrm{M}=0.3$ to $\mathrm{M}=0.9$.

The DLR-F17E high-speed model is a 1:2.653 geometrically scaled version of the DLR-F19 model. Its dimensions are shown in FIGURE 5. The moment reference point (MRP) is located at $(\mathrm{x} / \mathrm{y} / \mathrm{z})=$ $(0.234 \mathrm{~m} / 0.0 \mathrm{~m} / 0.0 \mathrm{~m})$. The suffix " $E$ " (for "intake", in German "Einlauf") was added to indicate that the model contains a modular center body section that can be exchanged with a flow-through inlet section. The effect of the inlet as well as various leading edge shapes have been studied in previous high-speed tests of the DLR F17E [11]-[13]

Just like the DLR-F19 the DLR-F17E is of modular set-up with non-adjustable control surface geometries. The trailing edge control surfaces were designed to replicate those of the DLR-F19 model as closely as possible. The hinge line of the considered control surfaces is located at $\mathrm{C}_{\text {ref }}=80 \%, 5 \%$ after the hinge line position of the DLR-F19. Additionally the span of the inboard control surfaces is approximately $13 \%$ shorter in comparison to the DLR-F19 inboard control surfaces. These geometrical changes could not be avoided due to manufacturing constraints.

In order to fix transition for the DLR-F17E it was chosen to prepare the leading edge using circular dots, both on the lower and upper surface. Dots of height $186 \mu \mathrm{m}$ were used. Their diameter and also the free space between two neighboring dots was about $1.25 \mathrm{~mm}$. In all high-speed tests the model was mounted on a rear sting (see FIGURE $6)$. 
The DLR-F17E is only equipped with a limited number of pressure ports, which are only used for $\mathrm{PSP}^{5}$ measurements. Like for the DLR-F19, an internal balance has been used to collect the force and moment data of the wind tunnel model configuration. A TASK balance, type MK XIV has been employed.

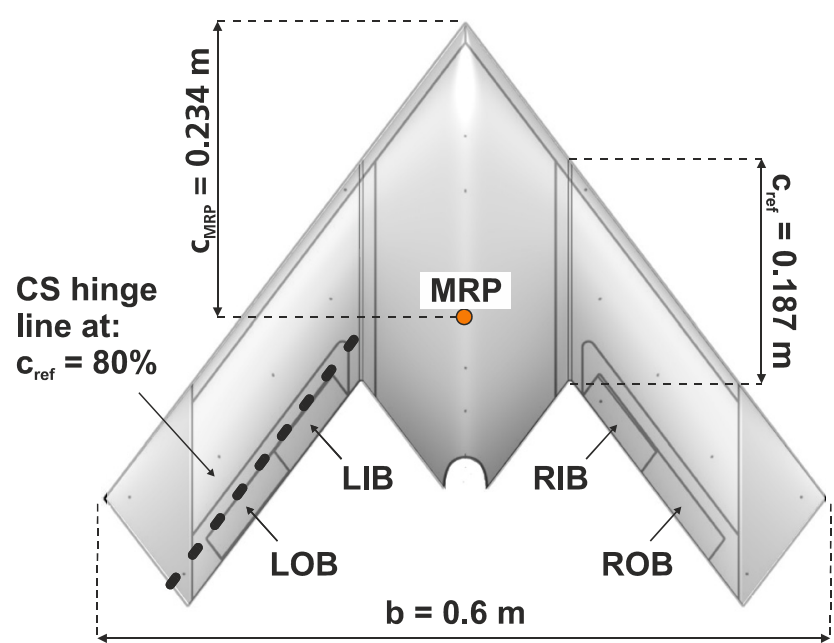

FIGURE 5. DLR-F17E model dimensions, and location of control surfaces.

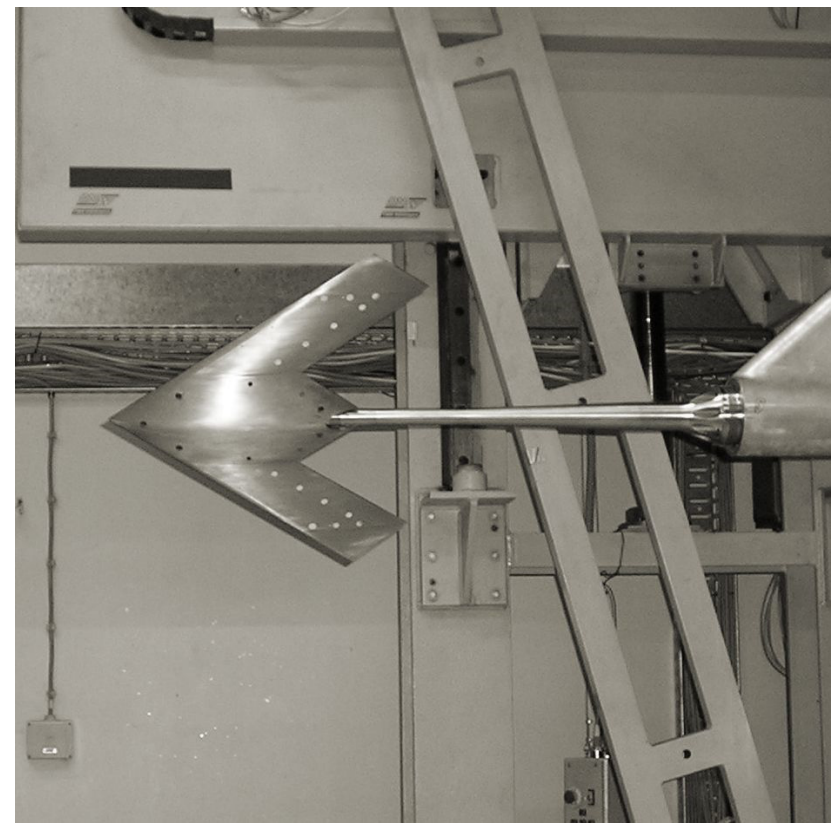

FIGURE 6. DLR-F17E model wind tunnel mounting arrangement of a back sting support in the DNW-TWG.

\section{RESULTS AND DISCUSSION}

This section will give an overview of the experimental outcome gathered with the low speed DLR-F19 model and the high speed DLR-F17E high speed model with control surface deflection. The axis convention can be found in FIGURE 1 and the convention of control surface deflections is shown in FIGURE 7.

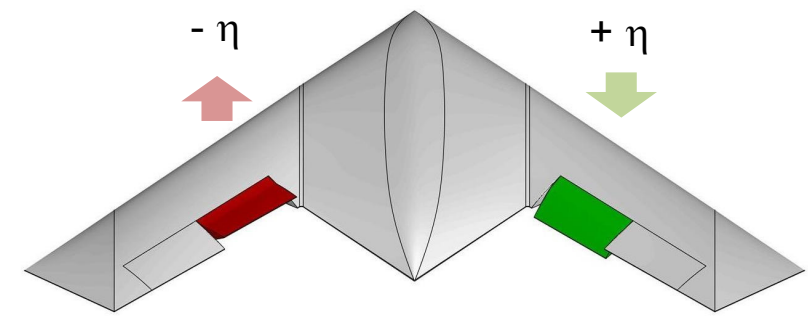

FIGURE 7. Control surface deflection convention for the DLR-F19 and DLR-F17E configuration.

\subsection{Mach number effect on the Baseline Configuration}

The effectiveness of control surfaces has been tested at low $(M=0.15)$ as well as high speeds $(M=0.5-0.9)$. FIGURE 8 illustrates the aerodynamic force and moment coefficients for the DLR-F19 and the DLR-F17E at various Mach numbers. In the following the comparison comprises a Mach number range of $\mathrm{M}=0.15-0.9$. A numerical comparability study of low and high speed experiments can be found in [14]. The two test Reynolds numbers could not be adjusted to match. However, the difference in Reynolds number can be regarded as small as the effect due to this Reynolds number is negligible at values as high as in the present tests [15].

The experimental tests in this range of Mach numbers reveal a strong effect of the Mach number (see FIGURE 8). With increasing Mach number, lift and drag coefficients are shifted to higher values. This is consistent with Prandtl-Glauert and Göthert rules.

Furthermore, locations of both, maxima and minimum, of the pitching moment are a function of the angle of attack. They move towards smaller angles when the Mach number increases. At $\mathrm{M}=0.15$ compressibility effects are irrelevant, however at $M \geq 0.5$ compressibility can no longer be neglected. Hence, the configuration experiences much stronger nose down pitch, represented by a larger dip in the curve. At the highest Mach number considered $(\mathrm{M}=0.9)$ qualitative changes can be observed, particularly, in the pitching moment and drag. At this Mach number oil flow and Schlieren images reveal the presence of a shock wave that extends across wings and fuselage already at angles of attack as low as $\alpha \approx 4.5^{\circ}[16]$.

\subsection{Static Low Speed Tests}

Throughout the low speed experiments a range of different design parameters were studied, such as control surface depth and different deflection angles. A detailed description of the outcome can be found in Huber et al. [17]. The core outcomes will however be stated within a subsection following the discussion of the static low speed tests selected here. The low speed results of the DLR-F19 will be incorporated into an aerodynamic database

\footnotetext{
${ }^{5}$ Pressure Sensitive Paint
} 
including also results of previous low speed wind tunnel tests with the SACCON wind tunnel model, conducted in different wind tunnel facilities. Model-to-model and tunnelto-tunnel repeatability as well as reproducibility was shown to be extraordinarily good up to an angle of attack of $\alpha=$ $17^{\circ}$, see Vicroy et al. [1], Huber et al. [17] and Vicroy et al. [18] and Huber et al [19].

\subsubsection{Single Sided Control Surface Deflection}

Within this subsection the effect of positive and negative, single sided control surface deflections at a Mach number of $\mathrm{M}=0.15$ will be discussed. Within this paper the focus lies on the moment changes due to the control surface deflection, in order to assess the controllability aspects in lateral- and longitudinal axis direction. A selected set of experimental results have been chosen for the discussion. FIGURE 9 and FIGURE 10 depict the aerodynamic coefficients of the low speed experimental test with the DLR-F19 for single sided positive and negative control surface deflections, respectively.

The positive (downward) deflection cases are deflected by $\eta=+20^{\circ}$ and the negative (upward) deflection cases are deflected by $\eta=-20^{\circ}$. A single inboard deflection will be considered as single deflection and a combined inboard and outboard control surface deflection will be considered as full span deflection from here onwards.

\subsubsection{Positive Control Surface Deflection}

Positive roll control is attempted by increasing the effective camber on one side due to the positive downward deflection of the control surfaces. The aerodynamic coefficients for this case are depicted in FIGURE 9.

The downward deflection gives rise to an overall increased lift and a slight increase in drag. The influence on the lift stays constant throughout the considered angle of attack range, whereas the influence on the drag is continuously increasing. A common issue for these types of tailless configurations is generating a sufficiently large side force and yawing moment to control the vehicle. When looking at the coefficients for side force and yaw the influence of the full-span positive control surface deflection is still very small and can be regarded as negligible. A clear need is thus given to undertake more investigations to find possible solutions for this limited yaw control effectiveness.

However, when considering the change in rolling moment due to control surface deflection it can be seen that the configuration experiences a considerable negative rolling moment when the inboard control surface, single deflection, on the right hand side is deflected downwards. The negative rolling moment is introduced as the right hand side of the configuration experiences an increase in lift, due to increased suction on the upper side of the configuration. The downward deflection of the control surface acts as an increase of camber. The negative rolling moment increases even further with additional deflection of the outboard control surface. The influence on the rolling moment is slightly decreasing with increasing angle of attack.

The incremental increase in negative rolling moment due to a full span deflection is smaller compared to the incremental increase in rolling moment due to the single inboard control surface deflection. The combined deflection of inboard and outboard control surface gives rise to a reduced influence of the outboard control surface. The inboard control surface diverts the approaching flow further towards the tip, the outboard control surface is hence subjected to a diverted flow running parallel to the hinge line, the influence decreases. Future investigations of single outboard control surfaces are being considered, in order to investigate possible summing up of powers. The existing results suggest, however, that at low speed, the reciprocal effects between control surface deflections hinder a simple adding up of single deflection influences to represent the effect of a full span deflection. The influence on the rolling moment for the full span deflection stays approximately constant with increasing angle of attack.

A positive control surface deflection also has a distinct influence on the pitching moment coefficient. The deflection gives rise to a shift of the pitching moment curve to lower positive values. Throughout the considered angle of attack the influence stays constant. The deflection gives rise to a change in moment balancing over the configuration along the x-direction. The downward deflection gives rise to an increased suction aft of the MRP, counteracting the nose up pitching moment generated by the configuration forward of the MRP. Increasing the deflection also affects the flow developing over the configuration. The deflection stabilizes the attached flow region present over the wing and supports an earlier development of the tip vortex.

\subsubsection{Negative Control Surface Deflection}

Positive roll control is attempted by means of lift destruction on one side due to the negative upward deflection of the control surfaces. The aerodynamic coefficients for this case are depicted in FIGURE 10.

The upward deflection gives rise to a reduced lift and, at lower angles of attack, to a slight increase in drag. The influence on the lift reduces slightly with increasing angle of attack, as does the influence on drag. For angles of attack larger than $10^{\circ}$ the influence on the drag is negligible. The influence on the side force as well as the yawing moment is even smaller than the influence already observed in the previous section. Hence these are considered as negligible.

When considering the change in rolling moment due to control surface deflection, it can be seen that the configuration experiences a negative rolling moment when the inboard control surface, single deflection, on the left hand side is deflected upwards. The negative rolling moment is introduced as the left hand side of the configuration experiences a reduction in lift, as the attached flow region is hindered to fully develop, due to the upward deflection. The negative rolling moment increases even further with additional deflection of the outboard control surface.

As already observed within the results from positive deflection, the incremental increase in negative rolling moment due to the additional deflection of the left outboard control surface of the configuration is smaller compared to the incremental influence of the single deflection when the angle of attack is small. However, for $\alpha>12^{\circ}$, the effect of the additional outer control surface becomes comparable or even greater than that of the only inner control surface. Due to the upward deflection of the control surface, the flow from the attached flow region is 
being diverted towards the tip and the outboard control surface sees flow parallel to the hinge line.

The pitching moment coefficient curve also shows distinct differences due to negative control surface deflection. The deflection gives rise to a shift of the pitching moment curve to higher positive values, compared to the baseline configuration. The nose up pitching moment is increased when applying a negative control surface deflection. Due to the negative deflection, the moment balancing over the configuration along the chord is changed. The upward deflection gives rise to a reduced lifting force aft the MRP. Further, the deflection also affects the vortex systems developing over the configuration. The deflection forces to the attached flow region present over the wing to divert towards the tip. This effect is more violent than the diversion due to downward deflection, and delays the development of the tip vortex.

\subsubsection{Combined Control Surface Deflection}

FIGURE 11 shows the effect of applying combined control surface deflections of opposite sign on both wing parts alongside the influences of the control surface deflections applied to the left and right and side separately. Here, the inboard as well as the outboard control surfaces on the left hand side are deflected by $\eta=-20^{\circ}$, denoted by $\eta_{\text {LHS }}$, and the inboard as well as the outboard control surfaces on the right hand side are deflected by $\eta=+20^{\circ}$, denoted by $\eta_{\text {RHS. }}$

Increased roll control is attempted by combining upward and downward deflections on opposing control surfaces. The double sided opposed full span deflection has barely an effect on the lift and drag coefficient, compared to the baseline configuration. Also for the combined case no real improvement in influence on the side force and yawing coefficients exist, the influence stays negligible.

When considering the change in rolling moment due to the combined control surface deflection, it can be seen that the configuration experiences a considerable increase in rolling moment compared to the single sided deflections. The combination of a lift enhancing downward deflection on the right hand side with a lift destructing upward deflection, on the left hand side, leads to the creation of a large negative rolling moment.

Contrary to the effect of single sided deflections, the pitching moment curve is not only shifted but the slope of the curve is changed, crossing the baseline pitching moment curve. The double sided opposed full span deflection gives rise to an increased nose up pitching moment in the lower angle of attack region up to $\alpha=5^{\circ}$; further increasing the angle of attack will give rise to a reduced nose up pitching moment.

\subsubsection{Additional Considered Parameters}

Within the low speed experiments also the influence of hinge line position (control surface depth), influence due to deflection angle and the effect of split flaps was considered [10]. Here the main outcomes were that an increase in control surface depth (moving the hinge line position from $\mathrm{c}_{\text {ref }}=0.80$ to 0.75 ) has no considerable influence on the aerodynamic coefficients; further, the overall influence of deflection angles of $\eta \leq 20^{\circ}$ on the flow is small, it can however be shown that the effect of positive deflections is approximately doubled when the amplitude of the deflection is increased by a factor of two. This outcome was also the driver to consider control surface deflections of $20^{\circ}$ for future experiments. Additionally, superposition effects were considered. It was found that adding one sided single deflections of the inboard control surfaces will reproduce the effects of a double sided inboard deflection. However, as soon as outboard deflections are involved, the superposition of single influences is not given. Due to the complex nature of the flow in the outer region of the configuration, the influence of the outboard control surfaces varies.

\subsection{Static High Speed Tests}

An equivalent number of control surfaces was considered for the high speed tests as there were for the low speed tests. Only experimental results of the DLR-F17E configuration incorporating single sided control surface deflections are considered in the following. Similar to the DLR-F19 low speed test, the DLR-F17E test results will be incorporated into the aerodynamic database. The configuration has also been subject to tests in various wind tunnel testing facilities. Tunnel-to-tunnel repeatability as well as reproducibility was shown to be extraordinarily good for the entire angle of attack range considered, see Rein et al. [15].

\subsubsection{Single Sided Control Surface Deflection}

Within this subsection the effect of positive and negative, single sided control surface deflections at a Mach number of $\mathrm{M}=0.8$ will be discussed. FIGURE 12 and FIGURE 13 depict the aerodynamic coefficients of the high speed experimental tests with the DLR-F17E for positive and negative control surface deflections, respectively. Positive deflections were applied on the right wing while negative deflections were applied on the left wing. In this manner changes in the rolling moment should have the same sign.

The positive (downward) deflection cases are deflected by $\eta=+10^{\circ}$ and the negative (upward) deflection cases are deflected by $\eta=-10^{\circ}$. Due to the greater kinetic energy in high speed flows control surface deflections have been limited to a maximum of $\eta=\left|10^{\circ}\right|$ already in the design phase of the DLR-F17E.

\subsubsection{Positive Control Surface Deflection}

FIGURE 12 depicts the influence on the aerodynamic coefficients of positive deflections of control surfaces on the right wing at $M=0.8$. Positive deflections at high speeds result in an increase of lift and drag, as already discussed in the low speed results. However, the influence on the lift and the drag is smaller than in the low speed results, as the control surfaces are only deflected by $\eta=$ $+10^{\circ}$. From experiments it was found that a deflection of the inner control surface is slightly more effective than that of the outer for all angles of attack considered.

Changes in side force and yawing moment can be regarded as negligibly small, staying within the margin of error of the balance, at least for angles of attack up to those corresponding with the dip in the pitching moment. At greater angles of attack the flow is no longer attached at the leading edge. This may result in an un-symmetric flow about the model and thus in lateral forces and moments. 
Rolling and pitching moments experience greater effects. A single sided increase of lift, caused by positive deflections on the right wing, results in a negative rolling moment. In the present high-speed tests the inner control surface turns out to be more efficient than the outer one, despite a smaller lever arm [20]. Therefore, the effect of combined deflections is only slightly greater than that of the inner control surface (see FIGURE 11). The reduced efficiency of the outer control surface is caused by changes of the flow field. Due to separation these are more pronounced at the outer part of the wing rendering the outer controls somewhat less effective. A similar observation was also made in the low-speed tests.

Finally, let us consider the pitching moment. A downward deflection produces a reduction in the pitching moment. Similar to the low speed results, the downward deflection increases the lift aft of the MRP, which results in a negative (nose down), contribution to the overall positive pitching moment.

\subsubsection{Negative Control Surface Deflection}

FIGURE 13 depicts the influence on the aerodynamic coefficients of negative deflections of control surfaces on the left wing, again at $M=0.8$. Negative deflections at high speeds have very little effect on lift and result in a small decrease of drag, as already discussed in the low speed results. However, the influence on the lift and the drag is smaller than in the low speed results, as the control surfaces are only deflected by $\eta=-10^{\circ}$.

As already noted in the low speed results and in the high speed results for positive deflections the changes in side force and yawing moment are negligibly small.

Both rolling and pitching moments are affected by upward deflections. As already seen in the low speed results, the negative deflection on the left hand side, mostly results in a negative rolling moment. Note, however, that in a small range at intermediate angles of attack the control power for the rolling moment becomes reversed, although only little. The same flow observations hold as already discussed in the low speed results. However, the difference between the outcomes for the only the inner and the combination of inner and outer control surfaces is small.

With upward deflections the pitching moment increases. Not surprisingly, the increase is greater for the combined deflection of both control surfaces. In an intermediate range of angles of attack, the inboard control surface shows almost no effect. These observations reflect the complex dependence of the vertical flow field on relatively small changes in the geometry.

\subsubsection{Additional Considered Parameters}

Within the high speed experiments the influence of deflection angles and split flaps was considered, as well as opposed single sided inboard and outboard deflections [20]. As already seen in the low speed results, the effect of positive deflections is approximately doubled when the amplitude is duplicated. In contrast to this, the effect of negative deflections on the rolling moment and pitching moment is about the same for intermediate angles of attack $\left(5<\alpha<10^{\circ}\right)$. It was shown that for positive and negative deflections, respectively, the resulting control powers exhibit an opposing behavior as do the coefficients; as long as the angle of attack stays below the value where the flow becomes fully separated. At greater angle of attack, the control power becomes irregular.

Generally, the control power of the inboard control surface is greater than that of the outboard control surface. At intermediate angles of attack $\left(\alpha \sim 5^{\circ}-10^{\circ}\right)$ the control power assumes a maximum for positive deflections, and a minimum for negative deflections. With increasing angle of attack the line of flow separation moves upstream exposing the control surfaces to more and more separated flow. Hence, their efficiency becomes reduced. In all cases the control power becomes again irregular at angles of attack beyond the one corresponding with the dip in the pitching moment, i.e., beyond the regime of attached flows when vortex breakdown may also play a role.

Additionally, superposition effects were considered, as in the low speed tests. It was found that, in a rough approximation, the control power of mixed control surface settings is given by a superposition of that of correspondingly deflected single control surfaces, similar to the effects observed with inboard control surfaces at low speed.

\section{CONCLUSION}

This report gave an overview of the experimental investigations conducted within the DLR internal projects FaUSST and Mephisto. The internal DLR research is closely connected to and coordinated with the research in the international research groups of the NATO STO/AVT. Within these projects a large amount of low- and high speed experimental data on a generic UCAV configuration was gathered to establish an experimental data set for focused CFD analysis and comparison. This experimental data in combination with the numerical results will be combined to form a hybrid database for aerodynamic replacement modelling.

The low speed experiments with the DLR-F19 configuration with control surfaces have shown that a control surface deflection of at least $\pm 20^{\circ}$ is needed to trigger definite effects on the flow and the resulting aerodynamic coefficients. Further, the first time control concept attempt lacks to produce sufficiently large moments in yaw. The deflections do however trigger sufficiently large moments on roll. The pitching moment is sensitive to the control surface deflection, as the deflection changes the moment balancing front and aft of the MRP. It was shown that superposition of single sided sole inboard deflection effects can reproduce double sided opposed inboard control surface deflections. Including outboard control surfaces the superposition possibility of single deflection influences is not given.

High speed tests of the DLR-F17E configuration with control surfaces have shown that the effect of the inboard control surface is comparable to that of the outboard surface, with the inboard control surface being slightly more efficient. At positive deflections and at angles of attack below the one corresponding with the dip in the pitching moment, the effect of the control surfaces increases approximately linearly with the angle of their deflection.

For low- as well as high speed test cases it has been 
shown that the behavior of the configuration becomes irregular at higher angles of attack where the onset of fully separated flows starts, and hence also the influence of the control surface deflections. In low speed flows this occurs at an angle of attack of $16^{\circ}$. In high speed flows the corresponding angle of attack varies between $\alpha \sim 8^{\circ}-15^{\circ}$, depending on the Mach number.

Often, the control power of two deflected control surfaces can be approximated as the sum of control powers of the two correspondingly deflected single deflection cases.

The examples presented in this paper have shown that the efficiency of the control devices of the present SACCON configuration need to be improved to provide more satisfying control power to the configuration. Steps in this directions, based on using unconventional control surfaces such as spoilers and smooth deformations of the wing tips have been considered numerically. First control concepts have already been tested in a low speed wind tunnel campaign [21].

\section{REFERENCES}

[1] Vicroy, D.D., Huber, K.C., Loeser, T.,and Rohlf, D., "Low-speed Dynamic Wind Tunnel Test Analysis of a Generic $53^{\circ}$ Swept UCAV Configuration with Controls," $32^{\text {nd }}$ AIAA Applied Aerodynamics Conference, AIAA-2014-2003, Atlanta, GA, June 2014.

[2] Schütte, A., Huber, K.C., Zimper, D., "Numerische Aerodynamische Analyse und Bewertung einer agilen hoch gepfeilten Flugzeugkonfiguration," Deutscher Luft- und Raumfahrtkongress, Rostock, 22. - 24. September 2015.

[3] Cummings, R.M., Schuette, A. et al., "Assessment of Stability and Control Prediction Methods for NATO Air and Sea Vehicles," NATO RTO/AVT, Final Report of the AVT-161 Task Group, RTO-TR-AVT-161, September 2012

[4] Rein, M., "Measurements of aerodynamic forces and moments on the DLR-F17E model in low- and highspeed flows," DLR Internal Report, DLR-IB 224-2011/ A61, DLR, Institute for Aerodynamics and Flow Technology, Göttingen 2011.

[5] Schütte, A., Hummel, D., and Hitzel, S.M., "Flow Physics Analyses of a Generic Unmanned Combat Aerial Vehicle Configuration," Journal of Aircraft, Vol. 49, Issue 6, pp. 1638 - 1651, November 2012.

[6] Schütte, A., Hummel, D., and Hitzel, S., "Numerical and Experimental Analyses of the Vortical Flow Around the SACCON Configuration", 28th AIAA Applied Aerodynamics Conference, AIAA-2010-4690, Chicago, IL, June 2010.

[7] Website of the German Dutch Wind Tunnels: http://www.dnw.aero/wind-tunnels/nwb.aspx [14 ${ }^{\text {th }}$ May 2014].

[8] Rein, M., Höhler, G., Schütte, A., Bergmann, A., and Loeser, T., "Ground-Based Simulation of Complex Maneuvers of a Delta-Wing Aircraft," Journal of Aircraft, Vol. 45, Issue 1, pp. 286-291, JanuaryFebruary 2008.

[9] Bergmann, A., Huebner, A., and Loeser, T., "Experimental and numerical research on the aerodynamics of unsteady moving aircraft, " Progress in Aerospace Sciences, Vol. 44, Issue 2, pp. 121-137, February 2008.
[10] Huber, K.C. et al., "Static and dynamic forces, moments and pressure distribution measurements on the DLR-F19 configuration," DLR Internal Report, IB 124-2014/908, Institut für Aerodynamik und Strömungstechnik, Braunschweig, 2014.

[11] Rein, M., Gardner, A. D., and Rütten, M., "Study of the influence of a highly integrated flow-through intake on compressible flows about the lambda wing configuration SACCON," $30^{\text {th }}$ AIAA Applied Aerodynamics Conference, AIAA-2012-3324, New Orleans, LA, June 2012.

[12] Rein, M., and Gardner, A. D., "Generic Lambda Wing Configuration in Compressible Flow: Effect of Highly Integrated Intakes," Journal of Aircraft, Vol. 52, Issue 3, pp. 972-980, May-June 2015.

[13] Rütten, M., Saalfeld, B., Rein, M., Künemund, J., and Saalfeld, S., "Numerical Flow Investigation of Morphing Leading Edges for the Enhancement of Maneuverability of Unmanned Combat Air Vehicles," $30^{\text {th }}$ AIAA Applied Aerodynamics Conference, AIAA2012-3326, New Orleans, LA, June 2012.

[14] Huber, K.C., "Numerical Investigation of the Aerodynamic Properties of a Flying Wing Configuration," DLR Internal Report, DLR-IB 1242010/4, DLR, Institute for Aerodynamics and Flow Technology, Braunschweig 2010.

[15] Rein, M., Irving, J., Rigby, G., and Birch, T., "High speed static experimental investigations to estimate control device effectiveness and S\&C capabilities," $32^{\text {nd }}$ AIAA Applied Aerodynamics Conference, AIAA2014-2004, Atlanta, GA, June 2014.

[16] Zimper, D., and Rein, M., "Experimental and numerical analysis of the transonic vortical flow over a generic lambda wing configuration," $32^{\text {nd }}$ AIAA Applied Aerodynamics Conference, AIAA-2014-2005, Atlanta, GA, June 2014.

[17] Huber, K., Vicroy, D.D., Schütte, A., and Hübner, A.R., "UCAV model design investigations and static low speed wind tunnel experiments to estimate control device effectiveness and S\&C capabilities," $32^{\text {nd }}$ AIAA Applied Aerodynamics Conference, AIAA-2014-2002, Atlanta, GA, June 2014.

[18] Vicroy, D.D., Loeser, T., and Schuette A., "Static and Forced-Oscillation Tests of a Generic Unmanned Combat Air Vehicle, "Journal of Aircraft, Vol. 49, Issue 6, pp. 1558-1583, November 2012.

[19] Huber, K. C., Schütte, A., and Rein, M., "Numerical Investigation of the Aerodynamic Properties of a Flying Wing Configuration," $30^{\text {th }}$ AIAA Applied Aerodynamics Conference, AIAA -2012-3325, 2012

[20] Rein, M., "Tests of the effectiveness of elevons and split flaps for the DLR-F17E model in high-speed flows," DLR Internal Report, DLR-IB 224-2013/A85, DLR, Institute for Aerodynamics and Flow Technology, Göttingen 2013.

[21] Paul, M. C., Rütten, M., and Rein, M., "Experimental and numerical investigations on unconventional control concepts for agile and highly swept aircraft configurations," Deutscher Luft- und Raumfahrtkongress, Rostock, 22. - 24. September 2015 


\section{APPENDIX}
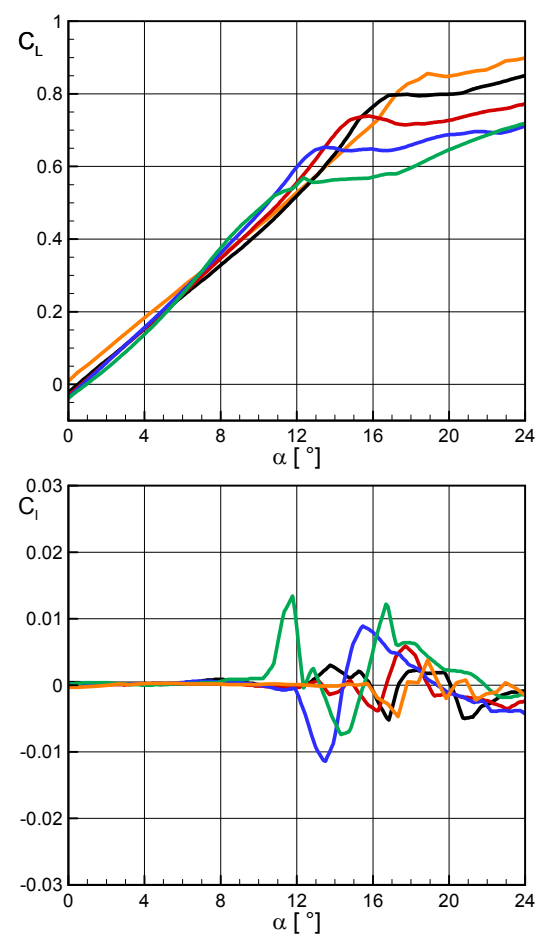
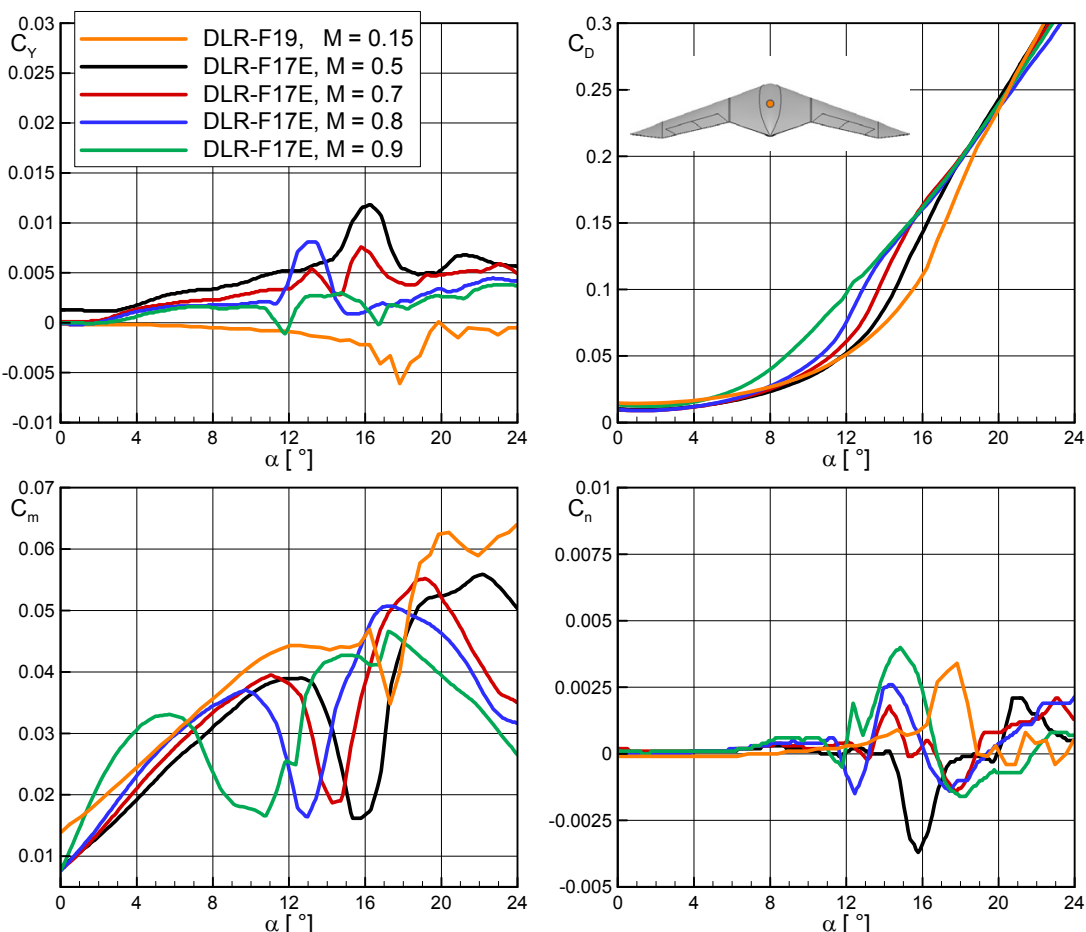

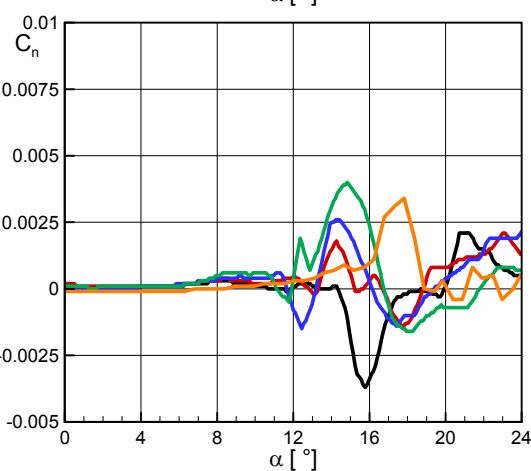

FIGURE 8. Effect of Mach number on the aerodynamic coefficients on the baseline configuration; low speed experimental results from the DLR-F19 baseline configuration $\left(M=0.15, R e=1.6 \cdot 10^{6}\right)$ in comparison with the high speed experimental results from the DLR-F17E baseline configuration $\left(M=[0.5,0.7,0.80 .9], \operatorname{Re}=\left[1.7,2.1,2.3,2.4 \cdot 10^{6}\right]\right)$.
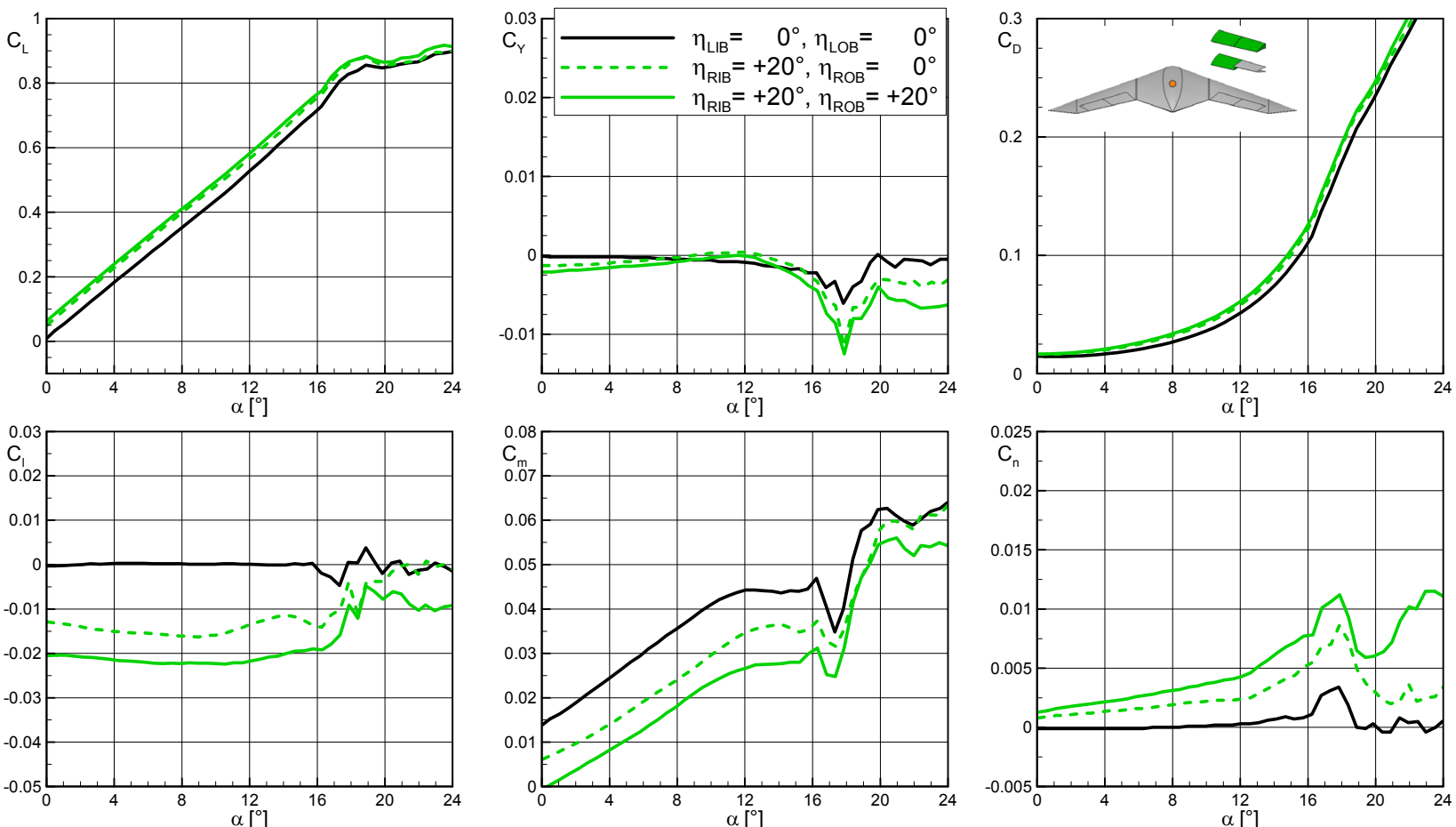

FIGURE 9. Effect of positive, downward, single sided control surface deflection on aerodynamic coefficients; low speed experimental results from the DLR-F19 $\left(M=0.15\right.$ and $\left.R e=1.6 \cdot 10^{6}\right)$. 

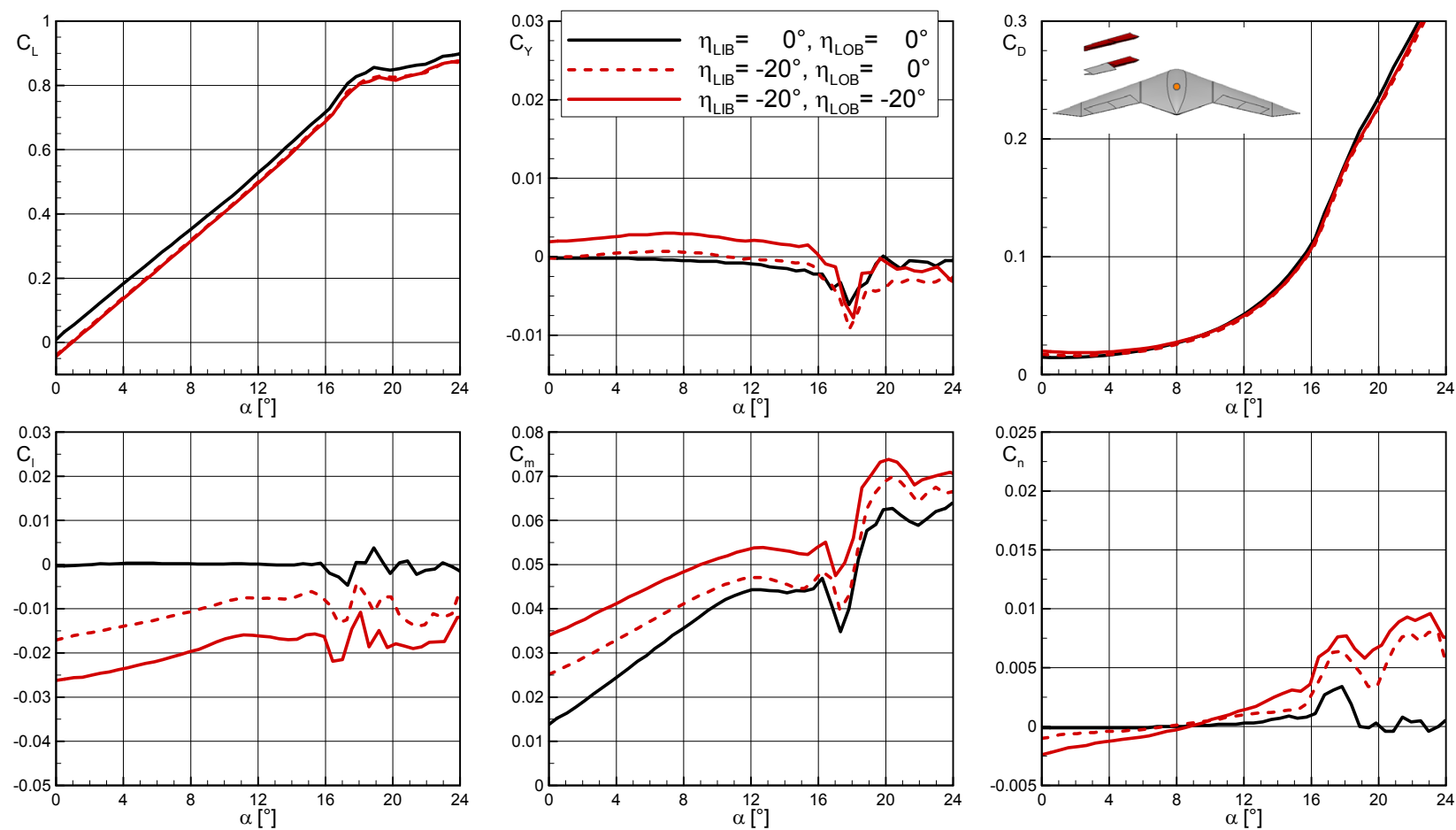

FIGURE 10. Effect of negative, upward, single sided control surface deflection on aerodynamic coefficients; low speed experimental results from the DLR-F19 $\left(M=0.15\right.$ and $\left.R e=1.6 \cdot 10^{6}\right)$.
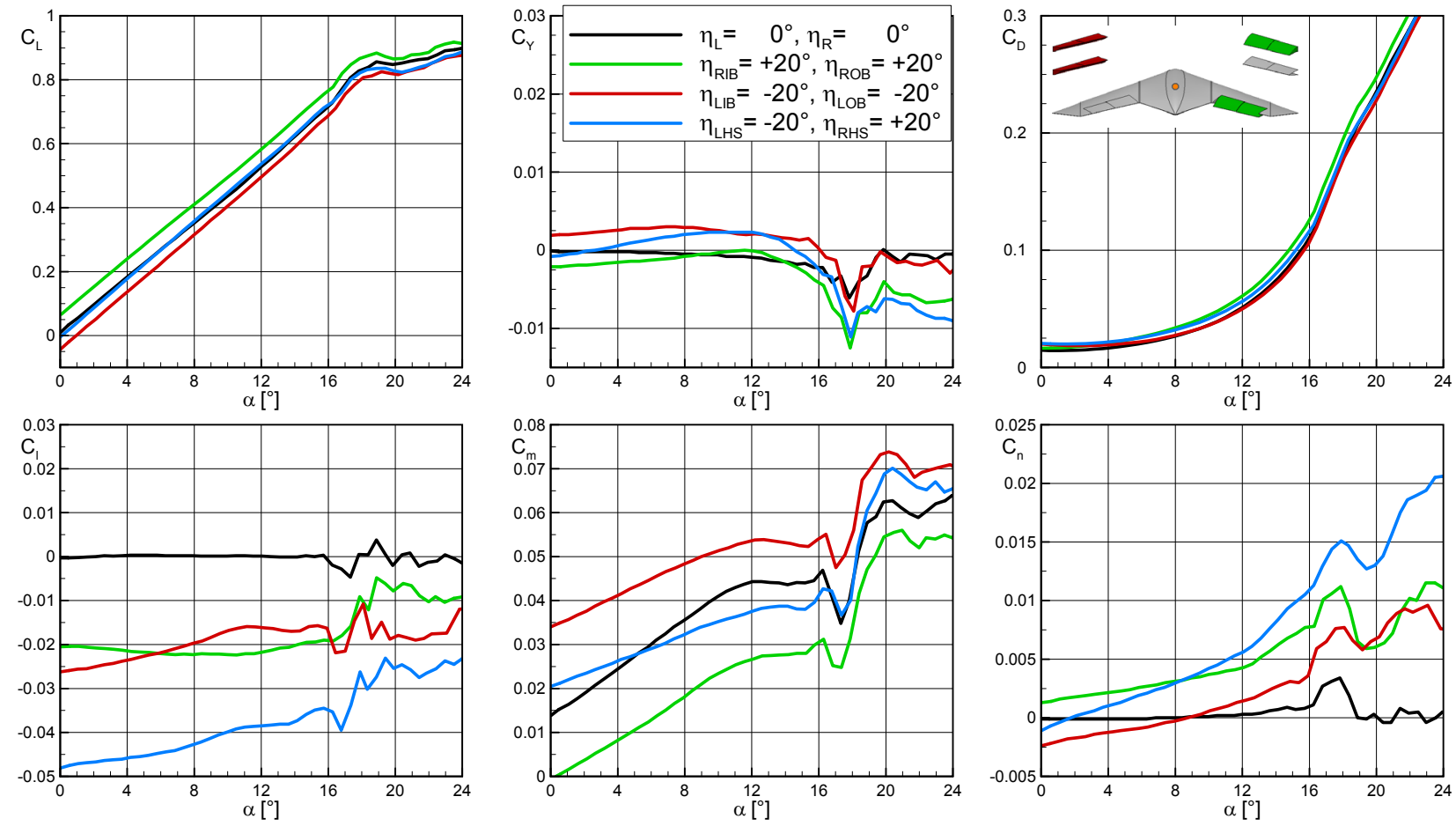

FIGURE 11. Effect of double sided opposed full span control surface deflection on aerodynamic coefficients; low speed experimental results from the DLR-F19 $\left(M=0.15\right.$ and $\left.R e=1.6 \cdot 10^{6}\right)$. 

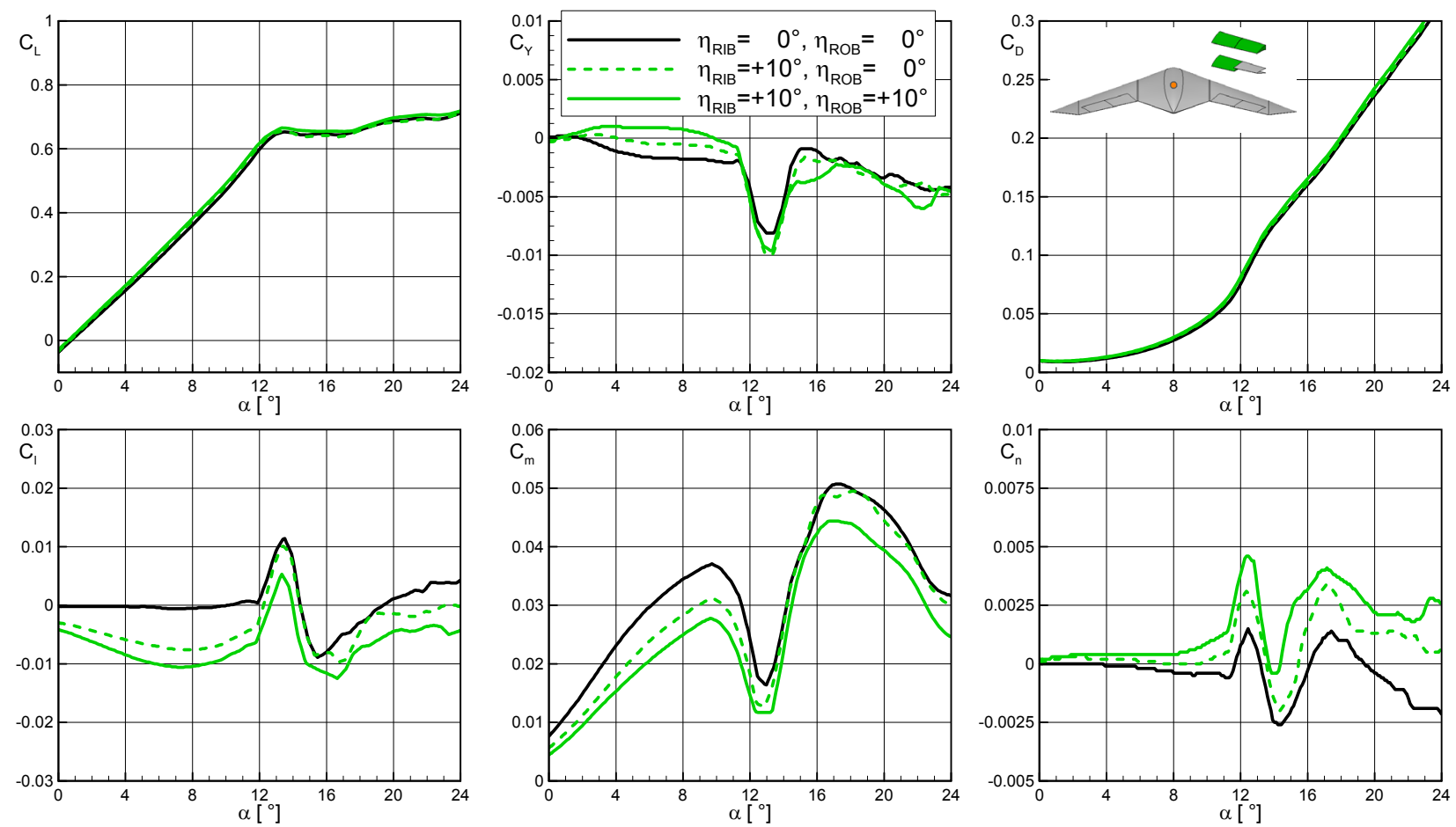

FIGURE 12. Effect of positive, downward, single sided control surface deflection on aerodynamic coefficients; high speed experimental results from the DLR-F17E $\left(M=0.8\right.$ and $\left.R e=2 \cdot 4 \cdot 10^{6}\right)$.
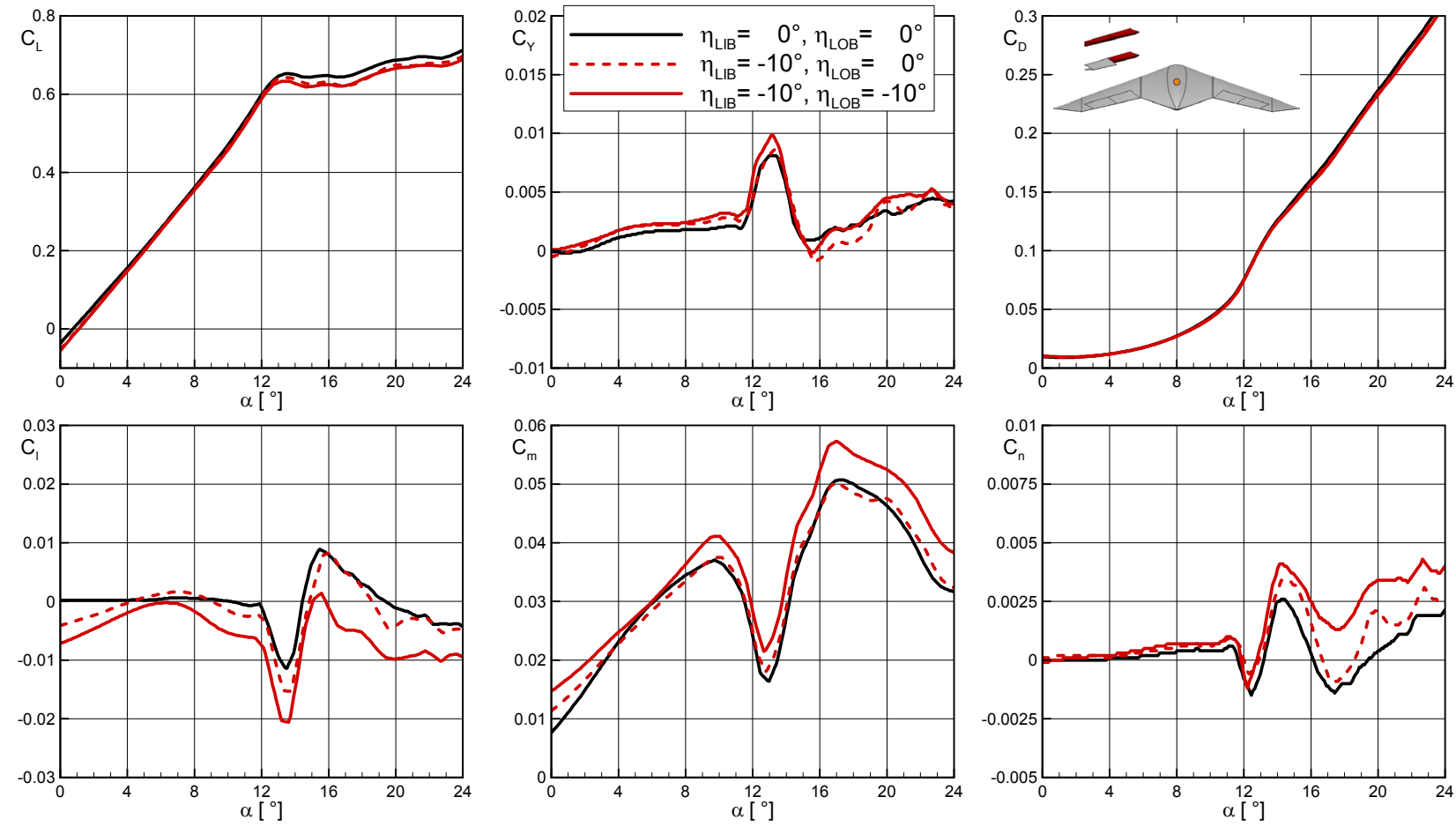

FIGURE 13. Effect of negative, upward, single sided control surface deflection on aerodynamic coefficients; high speed experimental results from the DLR-F17E $\left(M=0.8\right.$ and $\left.R e=2.4 \cdot 10^{6}\right)$. 